\title{
A New Perspective on Vertex Connectivity
}

\author{
Keren Censor-Hillel \\ Technion \\ Haifa, Israel \\ ckeren@cs.technion.ac.il
}

\author{
Mohsen Ghaffari \\ MIT \\ Cambridge, MA, USA \\ ghaffari@mit.edu
}

\author{
Fabian Kuhn \\ University of Freiburg \\ Freiburg, Germany \\ kuhnecs.uni-freiburg.de
}

\begin{abstract}
Edge connectivity and vertex connectivity are two fundamental concepts in graph theory. Although by now there is a good understanding of the structure of graphs based on their edge connectivity, our knowledge in the case of vertex connectivity is much more limited. An essential tool in capturing edge connectivity are edge-disjoint spanning trees. The famous results of Tutte and Nash-Williams show that a graph with edge connectivity $\lambda$ contains $\lfloor\lambda / 2\rfloor$ edge-disjoint spanning trees.

We present connected dominating set (CDS) partition and packing as tools that are analogous to edgedisjoint spanning trees and that help us to better grasp the structure of graphs based on their vertex connectivity. The objective of the CDS partition problem is to partition the nodes of a graph into as many connected dominating sets as possible. The CDS packing problem is the corresponding fractional relaxation, where CDSs are allowed to overlap as long as this is compensated by assigning appropriate weights. CDS partition and CDS packing can be viewed as the counterparts of the well-studied edge-disjoint spanning trees, focusing on vertex disjointedness rather than edge disjointness. We constructively show that every $k$-vertex-connected graph with $n$ nodes has a CDS packing of size $\Omega(k / \log n)$ and a CDS partition of size $\Omega\left(k / \log ^{5} n\right)$. We moreover prove that the $\Omega(k / \log n)$ CDS packing bound is existentially optimal.

CDS packing allows us to analyze vertex connectivity in the of context random vertex sampling. We show that if vertices of a $k$-vertex-connected graph are independently sampled with probability $p$, then the graph induced by the sampled vertices has vertex connectivity $\tilde{\Omega}\left(k p^{2}\right)$. This bound is optimal up to polylogarithmic factors.

As an additional application, we also show that CDS packings are tightly related to the (throughput of) store-and-forward algorithms in the networking model where in each time unit, each node can send one bounded-size message to all its neighbors. As a consequence, our $\Omega(k / \log n)$ CDS packing construction yields a store-and-forward broadcast algorithm with optimal throughput.
\end{abstract}




\section{Introduction and Related Work}

Vertex and edge connectivity are two core graph-theoretic concepts as they are basic measures for the robustness and throughput capacity of a graph. While by now a lot is known about edge connectivity and its connections to related graph-theoretic properties and problems, our knowledge about vertex connectivity is much scarcer and many related problems remain open.

As an example, given a graph $G$, assume that each edge or node is independently sampled with probability $p$. How large should $p$ be such that the subgraph given by the sampled edges or the one induced by the sampled nodes is connected (this is sometimes also known as the network reliability) or such that the sampled subgraph satisfies some other properties. Intuitively, the larger the connectivity of $G$ is, the smaller we should be able to choose $p$ such that the sampled subgraph remains connected. For edge connectivity and sampling edges, Lomonosov and Poleskii [22] verified this intuition already four decades ago: if $p=\Omega\left(\frac{\log n}{\lambda}\right)$, where $\lambda$ is the edge-connectivity of $G$, then the edge-sampled graph is connected with high probability and this threshold is optimal. In the special case of sampling edges of a complete graph, this corresponds to the $\frac{\ln n}{n}$ probability threshold for connectivity in the Erdős-Rényi random graph model. Karger [13] showed that assuming $p=\Omega\left(\frac{\log n}{\lambda}\right)$, the edge-connectivity of the edge-sampled graph will be around $\lambda p$, w.h.p., and in fact, for such $p$, the size of each edge cut remains around its expectation. In the following years, these results, and extensions thereof, have emerged as powerful tools, having implications for numerous important problems, see e.g. [3, 13, 15, 17]

In contrast, prior to our work, for vertex sampling (and vertex connectivity), even the most basic of these questions remained open. In the present paper, we prove results of the same flavor as the ones discussed above, but in the context of vertex sampling rather than edge sampling. In particular, we show that if each node of a $k$-vertex connected graph $G$ is independently sampled with probability $p$, then w.h.p., the graph induced by the sampled nodes has vertex connectivity at least $\tilde{\Omega}\left(k p^{2}\right)$. We also show that this is existentially tight up to log-factors 1

The main hurdle on the way to proving these results is that there can be an exponential number of "small" vertex cuts. When arguing that the subgraph induced by a randomly chosen subset of nodes is connected, one essentially needs to show that for each vertex cut of the graph, at least one node is selected. However, it has been shown that even the number of the minimum vertex cuts of a $k$-vertex connected graph can be as large as $\Theta\left(2^{k}(n / k)^{2}\right)$ [11]. Note that this is in stark contrast to the case of edge cuts, where the number of minimum edge cuts is known to be bounded by $O\left(n^{2}\right)$ and the number of edge cuts of size $\alpha \cdot \lambda$ in a graph with edge-connectivity $\lambda$ is at most $O\left(n^{2 \alpha}\right)[12,16]$. This $O\left(n^{2 \alpha}\right)$ bound is the main tool in studying edge cuts after random sampling. 2

Our main technical contribution is a method to decompose a graph into (almost) disjoint connected dominating sets (CDSs). We define a $C D S$ partition of size $K$ as a partition of the nodes of a graph into $K$ connected dominating sets. We also define CDS packings as a natural generalization of CDS partitions. A CDS packing is a collection of CDSs with positive weights such that for each node $v$, the sum of the weights of all CDSs containing $v$ is at most 1 . The size of a CDS packing is the total weight of all CDSs in the collection. We show that every $k$-vertex connected graph $G$ has a CDS packing of size at least $\Omega(k / \log n)$. We also show that this is optimal in the sense that for all $n$ and $k$, there are $k$-vertex connected $n$-node graphs for which the largest CDS packing has size at most $O(k / \log n)$. A generalized version of the CDS packing upper bound is the basis for the random sampling result mentioned above and also for a CDS partition upper bound showing that every $k$-vertex connected graph has a CDS partition of size at least $\Omega\left(k / \log ^{5} n\right)$.

CDS partitions and CDS packings can be seen as the "vertex world" analogues of the well-studied edgedisjoint spanning trees. Note that CDS partitions and CDS packings can equivalently be seen as collections of vertex disjoint dominating trees and dominating tree packings, respectively, by removing cycles after solving the problem. Edge-disjoint spanning trees have been a classical problem in graph theory (also studied under the title of decomposing a graph into connected factors), and they have numerous applications for

\footnotetext{
${ }^{1}$ For exact statements and a more extensive discussion of our results, we refer to Section 1.1

${ }^{2}$ We remark that the (tightest) proofs of this bound use the edge-disjoint spanning tree results of Tutte and Nash-Willams [16].
} 
different problems concerning edge-connectivity (see e.g., [13]). In a graph with edge connectivity $\lambda$, the size of a maximum spanning tree packing is clearly at most $\lambda$. Using the famous results of Tutte and NashWilliams [24]26], Kundu [19] showed that the size of a maximum set of edge-disjoint spanning trees is at least $\left\lfloor\frac{\lambda}{2}\right\rfloor$. Thus there is no asymptotic gap between the size of the best edge-disjoing spanning tree collection (or packing) and the edge connectivity. Our results show the corresponding relation between vertex connectivity and dominating tree packing and partition: the gap for dominating tree packing is $\Theta(\log n)$, whereas the gap for dominating tree partition is lower bounded by $\Omega(\log n)$ and upper bounded by $O\left(\log ^{5} n\right)$.

Apart from being interesting and natural structures of their own, CDS partition and CDS packing also have interesting applications in communication networks. Assume that in every time unit, each node of a network can locally broadcast a message of at most $B$ bits to all its neighbors. We show that the achievable total throughput when globally broadcasting messages using store-and-forward routing algorithm 3 can exactly be characterized by the size of the largest CDS packing of the network graph. As a consequence, we get that in $k$-vertex connected networks, such algorithms can achieve an optimal throughput of $\Theta(k / \log n)$ messages per round. Techniques of [7] show that network coding can achieve a throughput of $\Theta(k)$. Thus, our results imply that the network coding advantage for simultaneous broadcasts is $\Theta(\log n)$. Note that determining the network coding advantage for different communication models and problems is one of the important questions when studying network coding (see, e.g., [1, 6, 20, 21]).

\subsection{Results}

To cope with the problem of facing exponentially many small vertex cuts, we use a layering idea. For all our results, we need to find or prove the existence of a collection of small connected dominating sets of some graph $H$ with large vertex connectivity. Whereas in all cases, domination will be straightforward, obtaining connectivity is much more challenging. We assume that the nodes of $H$ are partitioned into $O(\log n)$ layers. We go through the layers one-by-one and establish connectivity by growing components as we proceed. Growing components turns out easier than proving connectivity directly and we can show that using this step-wise approach, it suffices to consider only polynomially many vertex cuts of $H$.

\section{CDS Packing and CDS Partition}

As described, our core technical contribution is an efficient algorithm to construct large CDS packings. As any connected dominating set of a graph $G$ must contain at least one node from every vertex cut of $G$, it is not hard to see that any CDS partition of a $k$-vertex connected graph has size at most $k$. Using a similar argument, it also follows that the largest CDS packing of a $k$-vertex connected graph has size at most $k$. Our main result shows that this basic upper bound can almost be achieved.

Theorem 1.1. Every $k$-vertex-connected $n$-node graph has a CDS packing of size $\Omega\left(\frac{k}{\log n}+1\right)$.

Specifically, we show how to construct a collection of $k$ CDSs, each consisting of $O\left(\frac{n \log n}{k}\right)$ nodes, such that each node is in at most $O(\log n)$ of the CDSs. Based on the CDS packing construction, we also obtain an efficient way to get a large CDS partition leading to the following result.

Theorem 1.2. Every $k$-vertex-connected n-node graph has a CDS partition of size $\Omega\left(\frac{k}{\log ^{5} n}+1\right)$.

In addition, we complement these results by showing that our $\Omega\left(\frac{k}{\log n}\right)$ CDS packing bound is asymptotically optimal in general.

Theorem 1.3. For any sufficiently large $n$, and any $k$, there exist $n$-node graphs with vertex connectivity $k \geq 1$ where the maximum CDS packing size, and thus also the maximum CDS partition size, are $O\left(\frac{k}{\log n}+1\right)$.

\footnotetext{
${ }^{3}$ Store-and-forward algorithms constitute the classical paradigm of message routing, where network nodes only forward the messages they receive from their neighbors, possibly with some changes in header information, without the use of network coding or any other form of combining different messages into new ones.
} 


\section{Vertex Connectivity and Random Sampling:}

As a specific application of (a generalized version of) our CDS packing construction, we obtain a tool to analyze the graph that is obtained when randomly sampling a subset of the nodes of a graph with large vertex connectivity. Mainly, we prove a lower bound on the vertext connectivity of the graph induced by a set of randomly sampled nodes. Note that in the following, for a graph $G=(V, E)$ and set of nodes $S \subseteq V, G[S]$ denotes the subgraph of $G$ induced by $S$.

Theorem 1.4. Consider a k-vertex-connected, n-node graph $G=(V, E)$ and let $S$ be a subset of $V$ where each node $v \in V$ is included in $S$ (i.e., sampled) independently with probability $p$. W.h.p., the graph $G[S]$ has vertex-connectivity $\Omega\left(\frac{k p^{2}}{\log ^{3} n}\right) \cdot 4$

A simple intuitive argument shows that up to logarithmic factors, the statement of Theorem 1.4 is the best possible. Consider a graph consisting of two cliques of size $k$, connected via a matching of $k$ edges. When randomly choosing each node with probability $p$, the vertex connectivity of the graph induced by the chosen nodes is given by the number of surviving matching edges. As each of these edges survives with probability $p^{2}$ and there are $k$ such edges, the expected vertex connectivity after sampling, even in this simple graph, is $k p^{2}$. Theorem 1.4 also gives a lower bound on $p$ such that the induced graph of the sampled nodes is connected, w.h.p. A slightly tighter bound for this specifc case is given by the following theorem.

Theorem 1.5. Consider a k-vertex-connected, $n$-node graph $G=(V, E)$ and let $S$ be a subset of $V$ where each node $v \in V$ is included in $S$ (i.e., sampled) independently with probability $p$. If $p>\alpha \frac{\log n}{\sqrt{k}}$ for $a$ sufficiently large constant $\alpha$, then $G[S]$ is connected, w.h.p.

We prove Theorem 1.5 in Section 3 Apart from being interesting by itself, we use this theorem to introduce the basic proof structure that we also use for our other, more involved results. The following observation shows that for most values of $k$, Theorem 1.5 is tight up to a factor of $O(\sqrt{\log n})$ (a formal argument appears in Appendix (C).

Observation 1.6. For every $k$ and $n$, there exists an n-node graph $G$ with vertex connectivity $k$ such that if we independently sample vertices with probability $p \leq \frac{\sqrt{\log (n / 2 k)}}{\sqrt{2 k}}$, then the subgraph induced by the sampled nodes is disconnected with probability at least $1 / 2$.

\subsection{Additional Related Work}

The domatic number of a graph is the size of the largest partition of a graph into dominating sets. In [5] it is shown that for graphs with minimum degree $\delta$, nodes can be partition into $(1-o(1))(\delta+1) / \ln n$ dominating sets efficiently. This implies a $(1+o(1)) \ln n$-approximation, which is shown to be best possible unless $\mathrm{NP} \subseteq \operatorname{DTIME}\left(n^{O(\log \log n)}\right)$. Further, Hedetniemi and Laskar [10] present an extensive collection of results revolving around dominating sets. The CDS partition problem was first introduced in [9] where the size of a maximum CDS partition of a graph $G$ is called the connected domatic number of $G$. Zelinka [27] shows a number of results about the connected domatic number; in particular, that it is upper bounded by the vertex connectivity. [8] shows that the connected domatic number of planar graphs is at most 4 and also describes some relations between the number of edges of a graph and its connected domatic number. Finally, [23] argues that a large CDS partition can be useful for balancing energy usage in wireless sensor networks.

\footnotetext{
${ }^{4}$ Note that the theorem requires $k=\Omega\left(\log ^{3} n\right)$ to be meaningful. Such a polylogarithmic lower bound on the vertex connectivity is necessary for all our statements to become non-trivial. We would like to point out that this is in all cases necessary as, e.g., shown by Observation 1.6
} 


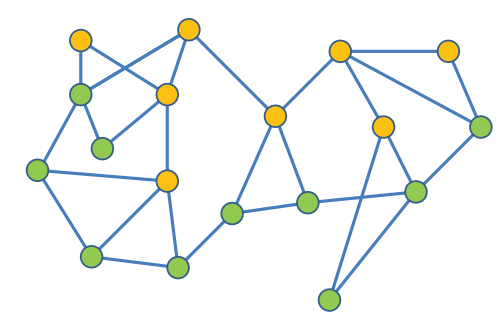

Figure 1: A CDS partition of size 2

\subsection{Roadmap}

The rest of the paper is organized as follows. In Section 2, we formally define CDS partition and packing and describe their connections to vertex connectivity, as well as their networking applications. In Section 3 , we present the result about the threshold on the vertex sampling probability for getting a connected induced subgraph. The analysis there demonstrate the main structure of our analysis for the later results in Sections 4 and 5. In Section 4, we present the CDS packing construction which is also used to derive our general bound on the vertex connectivity after vertex sampling. In Section 5 , we modify this construction to get the claimed CDS partition results. Finally, Section 6 presents some concluding remarks.

\section{Vertex Connectivity and Connected Dominating Sets}

In this section, we formally introduce CDS partitions and CDS packings. The structures can be seen as a wellorganized way to capture the vertex connectivity of a graph $G$. We therefore believe that they also provide a new perspective on vertex connectivity and related problems. The section is organized as follows. We start out by formally defining all necessary concepts. We then describe how CDS partition and CDS packing relate to vertex connectivity. Finally, we conclude the section by describing an application of the two structures in a networking context.

\subsection{Definitions}

Notations We usually work with a simple undirected graph $G=(V, E)$ as our main graph. Troughout, we use $n$ for the number of nodes of $G$. Also, for a subset $S \subseteq V$ of the nodes, we use $G[S]$ to denote the subgraph of $G$ induced by $S$.

Definition 2.1 (Dominating Set and Connected Dominating Set). Given a graph $G=(V, E)$, a set $S \subseteq V$ is called a dominating set iff each node $u \in V \backslash S$ has a neighbor in $S$. The set $S$ is called a connected dominating set $(C D S)$ iff $S$ is a dominating set and $G[S]$ is connected.

If $S$ is a dominating set of $G=(V, E)$, we also say that $S$ dominates $V$.

Definition 2.2 (CDS Partition). A CDS partition of a graph $G=(V, E)$ is a partition $V_{1} \cup \cdots \cup V_{t}=V$ of the nodes $V$ such that each set $V_{i}$ is a CDS. The size of a CDS partition is the number of CDSs of the partition. The maximum size of a $C D S$ partition of $G$ is denoted by $K_{C D S}(G)$.

Figure 1 presents a graph with a CDS partition of size 2, where nodes of each color form a CDS.

Definition 2.3 (CDS Packing). Let $C D S(G)$ be the set of all CDSs of a graph $G$. A CDS packing of G assigns a non-negative weight $x_{\tau}$ to each $\tau \in C D S(G)$ such that for each node $v \in V, \sum_{\tau \ni v} x_{\tau} \leq 1$. The size of this CDS packing is $\sum_{\tau \in C D S(G)} x_{\tau}$. The maximum size of a CDS packing of $G$ is denoted by $K_{C D S}^{\prime}(G)$. 
Note that a CDS partition is a special case of a CDS packing where each $x_{\tau} \in\{0,1\}$. In other words, CDS packing is the LP relaxation of CDS partition when formulating CDS partition as an integer programming problem in the natural way. Consequently, we have $K_{C D S}(G) \leq K_{C D S}^{\prime}(G)$ for every graph $G$.

We remark that the maximum CDS partition size of graph $G$ is sometimes also called the connected domatic number of $G[8,9,27]$. Analogously, the maximum CDS packing size can be referred to as the fractional connected domatic number of $G$.

\subsection{CDS Packing and Vertex Connectivity}

Menger's theorem tells us that a graph is $k$-vertex connected if and only if every pair of nodes $u$ and $v$ can be connected through $k$ internally vertex-disjoint paths. A CDS partition or packing produces analogous systems of paths, but with much stronger requirements: Given a CDS partition of size $t$, each pair of nodes $u$ and $v$ is connected by $t$ internally vertex-disjoint paths, one through each CDS. Consequently, we can choose the vertex-disjoint paths for different pairs in a consistent way in the following sense. The paths for all the pairs can be colored by $t$ colors such that, paths of different colors are internally vertex-disjoint and each node pair can be connected by one path from each color. A CDS packing provides a relaxed version of such a path system, where for each pair, paths are allowed to have vertex-overlaps but the weighted overlap in each node is bounded by one. These observations closely resemble edge-disjoint spanning trees (see e.g. [19, 24, 26]). A set of $t$ edge-disjoint spanning trees gives rise to a similar colored system of edge-disjoint (rather than internally vertex-disjoint) paths.

In light of the above discussion, it is natural to ask how the maximum CDS packing or partition sizes compare with the vertex connectivity of a graph. One direction of this relation is straightforward. Since for each vertex cut, each CDS has to contain at least one node of this cut, we have $K_{C D S}(G) \leq k$ [8, 27]. Based on the same basic argument, the same upper bound also applies to CDS packings (proof in Appendix $\mathrm{A}$ ):

Proposition 2.4. For each graph with vertex-connectivity $k$, we have $K_{C D S}(G) \leq K_{C D S}^{\prime}(G) \leq k$.

The other direction of the relation is more interesting. In the "edge world", the famous Tutte and NashWilliams result [24,26] shows that each graph with edge connectivity $\lambda$ contains at least $\lambda / 2$ edge-disjoint spanning trees, creating a colored system of edge-disjoint paths as explained above without asymptotic loss compared to Menger's Theorem. Our main result in this respect is Theorem 1.1, which proves a corresponding statement for the "vertex world" by showing that any $k$-vertex connected graph has a CDS packing of size $\Omega(k / \log n)$. Our proof of Theorem 1.1, presented in Section 4, directly leads to an efficient construction of a CDS packing of size $\Omega(k / \log n)$. As argued, CDS packings thus offer a new structured way of looking at vertex connectivity. Unlike for edge connectivity, the additional structure comes at a price as we loose an $O(\log n)$-factor, which we show to be unavoidable (in Theorem 1.3 ).

As a testament to the strength of the structure, in the proof of Theorem 1.4, we explain that the same general construction produces a CDS packing of size $\tilde{\Omega}\left(k p^{2}\right)$ when nodes are sampled with probability $p$. Up to logarithmic factors, this solves the open question about the vertex connectivity after sampling. The CDS packing acts as a witness for the vertex connectivity of the sampled subgraph, showing that it is $\tilde{\Omega}\left(k p^{2}\right)$.

Also, in Section 5 we show that a similar construction produces a CDS partition of size $\Omega\left(\frac{k}{\log ^{5} n}\right)$. We find it interesting that this CDS partition construction itself uses Theorem 1.4, i.e., the sampling result.

\subsection{CDS Packing vs. Throughput}

To conclude Section 2, we explain that CDS packing and CDS partition are relevant structures, also from a networking point of view, as they are closely related to the throughput of store-and-forward algorithms. A store-and-forward algorithm corresponds to the classical paradigm of routing (in contrast to network coding) where each node only stores and forwards packets and does not combine messages, or parts of them. Consider a synchronous network model where in each round (time unit), each node can send one message of size at most $B$ bits to all of its neighbors. Such a communication model is motivated, e.g., when considering wireless 
networks and working above the MAC layer, i.e., a local broadcast layer (see e.g. [18]). In the described communication model, CDS packing perfectly captures the throughput of store-and-forward algorithms for concurrent global broadcasts:

Theorem 2.5. A CDS packing with size t provides a store-and-forward backbone with broadcast throughput $\Omega(t)$ messages per round. Inversely, a store-and-forward broadcast algorithm with throughput $t$ messages per round induces a CDS packing of size at least $t$.

Here we provide a brief intuition. The formal proof of Theorem 2.5 is deferred to Appendix $\mathbb{B}$. For the first part, consider a CDS partition of size $t$. Then, $t$ different messages can be routed along different CDSs simultaneously and thus we can concurrently make progress for $t$ messages (throughput $t$ ). To achieve this using a CDS packing, each node $v$ is time-shared between the CDSs which go through $v$ such that the duration that $v$ works for each CDS $\tau$ is proportional to the weight $x_{\tau}$. For the second part, consider a storeand-forward broadcast algorithm with throughput $t$ and run it using a sufficiently large number of messages. For each message $m$, the set of nodes that forward $m$ forms a CDS (otherwise, $m$ would not reach all the nodes). Choosing the weight of each given CDS proportional to the number of messages that use this CDS induces a CDS packing of size $t$.

Given this connection, we get that our CDS packing result (Theorem 1.1) also gives a store-and-forward broadcast algorithm with optimal throughput. We remark that in the general formulation of CDS packings, each node might participate in arbitrarily many (in fact up to exponentially many) CDSs. This would make CDS packing inefficient from a practical point of view if the number of messages is small compared to the number of CDSs used. Fortunately, in our construction (cf., Theorem 1.1), each node only participates in $O(\log n)$ CDSs, which makes the CDS packing efficient even for a small number of messages.

\section{Vertex Connectivity Under Vertex Sampling: Connectivity Threshold}

In this section, we study the threshold for the vertex sampling probability such that the sampled graph remains connected. This allows us to demonstrate the main outline of our analysis technique in a simple and clean way. In the next section, we extend the technique to prove our general bounds on CDS packing and on the vertex connectivity after sampling.

Theorem 1.5. (restated) Consider an arbitrary graph $G=(V, E)$ with vertex connectivity $k$. Let $S$ be a subset of $V$ where each node $v \in V$ is included in $S$ (i.e., sampled) independently with probability $p$. If $p>\alpha \frac{\log n}{\sqrt{k}}$ for a sufficiently large constant $\alpha$, then, w.h.p., $S$ is a CDS of $G$ and thus, $G[S]$ is connected.

Before proceeding with the actual proof of Theorem 1.5, we need to introduce a few basic concepts. As a key feature of our analysis, instead of trying to argue about the structure of the set of all the sampled nodes at once, we turn the sampling into a more evolving process, using a simple layering idea. To show that a set of randomly sampled nodes of a graph forms a CDS, we partition the nodes of the graph into $L$ layers and study how the sampled structure evolves when going through the layers one-by-one. In order for our arguments to work, we require the subgraph induced by each layer to have large vertex connectivity. This is hard to achieve when partitioning the nodes of the original graph $G$ into layers. Instead of arguing directly about $G$, we therefore analyze the sampling process for a graph $\mathcal{G}=(\mathcal{V}, \mathcal{E})$ that we call the virtual graph 5 and which is defined as follows. For each real node $v \in V$ (and a sufficiently large constant $\lambda$ ), create $L=\lambda \log n$ copies of $v$, one for each layer $\ell$ in $[1, L]$. Connect two virtual nodes if and only if they are copies of the same real node or copies of two adjacent real nodes. Note that for each layer $\ell$, the virtual nodes of layer $\ell$ induce a copy of $G$. For each set of virtual nodes $\mathcal{W} \subseteq \mathcal{V}$, we define the projection $\Psi(\mathcal{W})$ of $\mathcal{W}$ onto $G$ as the set $W \subseteq V$ of real nodes $w$, for which at least one virtual copy of $w$ is in $\mathcal{W}$. Note that two nodes in $\mathcal{G}$ are connected

\footnotetext{
${ }^{5}$ We remark here that this transformation to virtual graph $\mathcal{G}$ is not necessary in Section 3 but it is needed in Section 4 . In order to make the analysis of the two sections consistent and to get the readers used to the related notations before going into the complications of Section 4, we choose to use $\mathcal{G}$ in this section as well.
} 
if and only if their projections are connected in $G$ or if they project to the same node, implying that $\mathcal{G}[\mathcal{W}]$ is connected (or dominating) if and only if $G[\Psi(\mathcal{W})]$ is connected (or dominating).

To translate the sampling to $\mathcal{G}$, we use a simple coupling argument. Consider the following process: sample each virtual node with probability $q=1-(1-p)^{1 / L}$ and then sample each real node $v \in V$ if and only if at least one of its virtual copies is sampled (i.e., the sampled real nodes are obtained by projecting the sampled virtual nodes onto $G$ ). The probability of each real node being sampled is $1-(1-q)^{L}=p$. Henceforth, we work on $\mathcal{G}$ assuming that each virtual node is sampled independently with probability $q=$ $1-(1-p)^{1 / L} \geq \frac{p}{2 L}$. Let $\mathcal{V}_{\ell}$ be the set of all virtual nodes in layers $1, \ldots, \ell$ and let $\mathcal{S}_{\ell}$ be the set of all sampled virtual nodes from $\mathcal{V}_{\ell}$. We define $N_{\ell}$ to be the number of connected components of $\mathcal{G}\left[\mathcal{S}_{\ell}\right]$ and let $M_{\ell}=N_{\ell}-1$ (i.e., the excess number of components).

Proof Outline : We first show that the sampled virtual nodes of the first $L / 2$ layers already give domination. Then, for each layer $\ell \geq L / 2$, we look at components of $\mathcal{G}\left[\mathcal{S}_{\ell}\right]$ and show that adding sampled virtual nodes of layer $\ell+1$ merges (in expectation) a constant fraction of these components, each with at least one other component, while not creating new components. Consequently, we get that $M_{\ell}$ decreases essentially exponentially with $\ell$, until it becomes zero, at which point connectivity is attained. Formally, we use the following two key lemmas.

Lemma 3.1 (Domination Lemma). With high probability, $\mathcal{S}_{L / 2}$ dominates $\mathcal{V}$.

The proof of the Domination Lemma is straightforward and thus deferred to Appendix $\mathrm{C}$. In the following, let $\mathcal{D}$ be the set of dominating sets of $\mathcal{G}$ consisting only of nodes from layers $1, \ldots, L / 2$. Further, for all $\ell \geq L / 2$, let $\mathcal{D}_{\ell}$ contain all sets $\mathcal{T} \subseteq \mathcal{V}_{\ell}$ such that there exists a $D \in \mathcal{D}$ for which $D \subseteq \mathcal{T}$. That is, $\mathcal{D}_{\ell}$ is the collection of all sets of virtual nodes in layers $1, \ldots, \ell$ that contain a dominating set $D \in \mathcal{D}$. Note that using this notation, Lemma 3.1 states that $S_{L / 2} \in \mathcal{D}$, w.h.p.

Lemma 3.2 (Fast Merger Lemma). If $\mathcal{S}_{L / 2} \in \mathcal{D}$, then for each $\ell \geq \frac{L}{2}$, we have $M_{\ell+1} \leq M_{\ell}$. Moreover, for every $\ell \geq \frac{L}{2}$ and every $\mathcal{T} \in \mathcal{D}_{\ell}$, we have $\operatorname{Pr}\left[M_{\ell+1} \leq \frac{9}{10} \cdot M_{\ell} \mid \mathcal{S}_{\ell}=\mathcal{T}\right] \geq 1 / 3$.

Thus, as long as $\mathcal{S}_{L / 2}$ is a dominating set, the probability for reducing the number of components by a constant factor is at least $1 / 3$, independently of the result of the sampling in layers $1, \ldots, \ell$.

Connector Paths: To prove the Fast Merger Lemma, we define connector paths. Consider the transition from $\mathcal{G}\left[\mathcal{S}_{\ell}\right]$ to $\mathcal{G}\left[\mathcal{S}_{\ell+1}\right]$, suppose $N_{\ell} \geq 2$, and let $\mathcal{C}$ be a connected component of $\mathcal{G}\left[\mathcal{S}_{\ell}\right]$. Consider the projection $\Psi\left(\mathcal{S}_{\ell}\right)$ onto $G$. We say that a path $P$ of $G$ is a potential connector path for component $\Psi(\mathcal{C})$ if the following two conditions hold: (A) $P$ has one endpoint in $\Psi(\mathcal{C})$ and another endpoint in $\Psi\left(\mathcal{S}_{\ell} \backslash \mathcal{C}\right)$ and (B) $P$ has at most 2 internal nodes. From $P$ we derive a potential connector path $\mathcal{P}$ of $\mathcal{C}$ in $\mathcal{G}$ by taking the layer $\ell+1$ copy in $\mathcal{G}$ of the internal nodes of $P$, and for each endpoint $w$ of $P$ we take its copy in $\mathcal{S}_{\ell}$. We say that a potential connector path $\mathcal{P}$ is a connector path if and only if in the layer $\ell+1$ copy of $G$, the internal (virtual) nodes of $\mathcal{P}$ are all sampled. Note that if $\mathcal{C}$ has a connector path to another component $\mathcal{C}^{\prime}$, then in $\mathcal{G}\left[\mathcal{S}_{\ell+1}\right]$, the components $\mathcal{C}$ and $\mathcal{C}^{\prime}$ are merged with each other. Given this, in order to prove the Fast Merger Lemma, we show Lemma 3.3 and prove it. Given Lemma 3.3, we can prove Lemma 3.2 and consequently Theorem 1.5 using standard probability arguments. The details of those parts are deferred to Appendix C.

Lemma 3.3. For each $\ell \in[L / 2, L-1]$, and every $\mathcal{T} \in \mathcal{D}_{\ell}$, if $\mathcal{S}_{\ell}=\mathcal{T}$ and $N_{\ell} \geq 2$, then for each connected component $\mathcal{C}$ of $\mathcal{G}\left[\mathcal{S}_{\ell}\right]$, with probability at least $1 / 2, \mathcal{C}$ has at least one connector path.

Proof of Lemma 3.3. Fix a layer $\ell \in[L / 2, L-1]$, and an arbitrary set $\mathcal{T} \in \mathcal{D}_{\ell}$ and fix $\mathcal{S}_{\ell}=\mathcal{T}$. Consider the projection $\Psi\left(\mathcal{S}_{\ell}\right)$ onto $G$ and recall Menger's theorem: Between any pair $(u, v)$ of non-adjacent nodes of a $k$-vertex connected graph, there are $k$ internally vertex-disjoint paths connecting $u$ and $v$. Applying Menger's theorem to a node in $\Psi(\mathcal{C})$ and a node in $\Psi\left(\mathcal{S}_{\ell} \backslash \mathcal{C}\right)$, we obtain at least $k$ internally vertex-disjoint paths between $\Psi(\mathcal{C})$ and $\Psi\left(\mathcal{S}_{\ell} \backslash \mathcal{C}\right)$ in $G$. We first show that these paths can be shortened so that each of them has 
at most 2 internal nodes i.e., to get property (B) of potential connector paths. Pick an arbitrary one of these $k$ paths and denote it $P=v_{1}, v_{2}, \ldots, v_{r}$, where $v_{1} \in \Psi(\mathcal{C})$ and $v_{r} \in \Psi\left(\mathcal{S}_{\ell} \backslash \mathcal{C}\right)$. By the assumption that $\mathcal{S}_{L / 2}$ dominates $\mathcal{G}$, we get that $\Psi\left(\mathcal{S}_{\ell}\right)$ dominates $G$. Hence, since $v_{1} \in \Psi(\mathcal{C})$ and $v_{r} \in \Psi\left(\mathcal{S}_{\ell} \backslash \mathcal{C}\right)$, either there is a node $v_{i}$ along $P$ that is connected to both $\Psi(\mathcal{C})$ and $\Psi\left(\mathcal{S}_{\ell} \backslash \mathcal{C}\right)$, or there must exist two consecutive nodes $v_{i}$, $v_{i+1}$ along $P$, such that one of them is connected to $\Psi(\mathcal{C})$ and the other is connected to $\Psi\left(\mathcal{S}_{\ell} \backslash \mathcal{C}\right)$. In either case, we can derive a new path $P^{\prime}$ which satisfies (B) and is internally vertex-disjoint from the other $k-1$ paths since its internal nodes are a subset of the internal nodes of $P$ and are not in $\Psi\left(\mathcal{S}_{\ell}\right)$. After shortening all the $k$ internally vertex-disjoint paths, we get $k$ internally vertex-disjoint paths satisfying (A) and (B), i.e., $k$ internally vertex-disjoint potential connectors paths for $\Psi(\mathcal{C})$.

For each potential connector path $P$ of these $k$ vertex-disjoint potential connector paths, each virtual internal node of its copy $\mathcal{P}$ in layer $\ell+1$ of $G$ is sampled with probability $q$. Since each potential connector path $P$ has at most 2 internal nodes, the probability that all internal virtual nodes of its copy $\mathcal{P}$ in layer $\ell+1$ are sampled is at least $q^{2}=\left(\frac{p}{2 L}\right)^{2} \geq\left(\frac{\alpha}{2 \lambda}\right)^{2} \cdot \frac{1}{k}$. Hence, the expected number of connector paths is at least $\left(\frac{\alpha}{\lambda}\right)^{2}$. Because the $k$ potential connector paths are internally vertex-disjoint, the events of their copies being connector paths (having their internal virtual nodes in layer $\ell+1$ sampled) are independent. Therefore, choosing a large enough constant $\alpha$, we get that with probability at least $1 / 2$, component $\mathcal{C}$ has at least one connector path.

\section{Construction of CDS Packing, and Vertex Connectivity After Sampling}

In this section, we present our main CDS packing result.

Theorem 1.1. (restated) Any graph with vertex connectivity $k \geq 1$ has a CDS packing of size $\Omega\left(\frac{k}{\log n}+1\right)$.

We complement the above lower bound by an upper bound on CDS packing, showing that Theorem 1.1 is existentially optimal and loosing the $\Theta(\log n)$ factor is unavoidable. The proof of the following Theorem 1.3 , which is deferred to Appendix A, builds on a base graph $\mathrm{H}$, which has vertex connectivity $O(\log n)$ and maximum CDS packing size $O(1)$. It then uses the probabilistic method [2] to show that for any value of $k$, there exists a subgraph of $\mathrm{H}$ which shows the claimed logarithmic gap.

Theorem 1.3. (restated and rephrased) For any sufficiently large $n$ and any $k \in[1, n / 4]$, there exists a $k$-vertex-connected, n-node graph $G$ such that the maximum $C D S$ packing of $G$ is of size $O\left(\frac{k}{\log n}+1\right)$.

Our proof of Theorem 1.1 is constructive and it also shows that a CDS packing of size $O(k / \log n)$ can be found efficiently. Even more generally, the same construction allows to create a CDS packing of $G$ of size $\Omega\left(k p^{2} / \log ^{3} n\right)$, if nodes are independently sampled with probability $p$ and the CDSs of the packing are restricted to only consist of sampled nodes. Using Proposition 2.4, this CDS packing acts as a witness to the vertex connectivity of the sampled graph and it thus proves our main sampling theorem.

Theorem 1.4. (restated) Consider an arbitrary graph $G=(V, E)$ with vertex connectivity $k$. Let $S$ be a subset of $V$ where each node $v \in V$ is included in $S$ (i.e., sampled) independently with probability p. W.h.p., the graph $G[S]$ has a CDS packing of size $\Omega\left(\frac{k p^{2}}{\log ^{3} n}\right)$, and thus, also vertex connectivity $\Omega\left(\frac{k p^{2}}{\log ^{3} n}\right)$.

To capture Theorems 1.1 and 1.4 together, in the remainder of this section, we discuss the construction in the case of vertex sampling with probability $p$ and give a CDS packing with size $\Omega\left(k q^{2} / \log n\right)$, where $q=1-(1-p)^{1 /(3 L)}$ and $L=\lambda \log n=\Theta(\log n)$ is the number of layers (as in Section 3). Since $q \geq \frac{p}{6 L}=\Theta\left(\frac{p}{\log n}\right)$, the CDS packing size is $\Omega\left(k q^{2} / \log n\right)=\Omega\left(k p^{2} / \log ^{3} n\right)$, thus proving Theorem 1.4. When we set $p=1$ (i.e., no sampling), we get $q=1$ and thus, the CDS packing size becomes $\Omega(k / \log n)$ as claimed by Theorem 1.1 . 


\subsection{Construction of the CDS Packing}

To construct the claimed CDS packing, similarly to Section 3, we transform the graph $G=(V, E)$ into a virtual graph $\mathcal{G}=(\mathcal{V}, \mathcal{E})$, this time consisting of $3 L$ copies of $G$. As in Section 3, to translate the sampling to $\mathcal{G}$, we sample each virtual node with probability $q=1-(1-p)^{1 /(3 L)} \geq \frac{p}{6 L}$. To construct the promised CDS packing on the sampled real nodes, we create a CDS partition of size $\Omega\left(k q^{2}\right)=\Omega\left(k p^{2} / \log ^{2} n\right)$ on the sampled virtual nodes. Since each real node has $\Theta(\log n)$ virtual copies, by giving a weight of $1 / \Theta(\log n)$ to each CDS, we directly get the claimed CDS packing.

In the rest of this section, we work on $\mathcal{G}$ and show how to construct $t=\delta \cdot k q^{2}$ vertex-disjoint connected dominating sets on the sampled virtual nodes, for a sufficiently small constant $\delta>0$. We have $t$ classes and we assign each sampled virtual node to one class, such that, eventually each class is a CDS, w.h.p. To organize the construction, we group the virtual nodes in $L$ layers, putting three copies of graph $G$ in each layer. Inside each layer, the three copies are distinguished by a type number in $\{1,2,3\}$.

We use notations (partially) similar to Section 3 . Let $\mathcal{S}_{\ell}^{i}$ be the set of sampled nodes of layers 1 to $\ell$ that are assigned to class $i$. Let $N_{\ell}^{i}$ be the number of connected components of $\mathcal{G}\left[\mathcal{S}_{\ell}^{i}\right]$. Finally, define $M_{\ell}:=\sum_{i=1}^{t}\left(N_{\ell}^{i}-1\right)$ to be the total number of excess components after considering layers $1, \ldots, \ell$. Initially $M_{1} \leq n-t$, and as soon as $M_{\ell}=0$, each class induces a connected sub-graph. Thus, the goal will be to assign sampled virtual nodes to classes such that $M_{L}=0$.

The class assignments are performed in a recursive manner based on the layer numbers. We begin the assignment with a jump-start, assigning sampled virtual nodes of layers 1 to $\frac{L}{2}$ to random classes. We show in Lemma 4.1 that this already gives domination. The proof is deferred to Appendix $D$.

Lemma 4.1 (Domination Lemma). W.h.p., for each class $i, \mathcal{S}_{L / 2}^{i}$ dominates $\mathcal{V}$.

Note that the domination of each class follows directly from this lemma. For the rest of this section, we assume that for each class $i, \mathcal{S}_{L / 2}^{i}$ dominates $\mathcal{V}$, and we will use this property to get short connector paths.

After the first $\frac{L}{2}$ layers, we go over the layers one by one and for each layer $\ell \in[L / 2, L-1]$, we assign nodes of layer $\ell+1$ to classes based on the assignments of nodes of layers 1 to $\ell$. In the rest of this section, we explain this assignment for layer $\ell+1$. We refer to nodes of layers 1 to $\ell$ as old nodes whereas nodes of layer $\ell+1$ are called new nodes. The goal is to perform the class assignment of the new sampled nodes such that (in expectation) the number of connected components decreases by a constant factor in each layer, i.e., to get a Fast Merger Lemma. During the recursive assignments, our main tool will be a modified variant of the concept of connector paths, which we define next.

\subsection{Connector Paths}

Consider a class $i$, suppose $N_{\ell}^{i} \geq 2$, and consider a component $\mathcal{C}$ of $\mathcal{G}\left[\mathcal{S}_{\ell}^{i}\right]$. We use the projection $\Psi\left(\mathcal{S}_{\ell}^{i}\right)$ onto graph $G$ as defined in Section 3. A path $P$ in $G$ is called a potential connector for $\Psi(\mathcal{C})$ if it satisfies the following three conditions: (A) $P$ has one endpoint in $\Psi(\mathcal{C})$ and the other endpoint in $\Psi\left(\mathcal{S}_{\ell}^{i} \backslash \mathcal{C}\right),($ B) $P$ has at most two internal nodes, (C) if $P$ has exactly two internal nodes and has the form $s, u, w, t$ where $s \in \Psi(\mathcal{C})$ and $t \in \Psi\left(\mathcal{S}_{\ell}^{i} \backslash \mathcal{C}\right)$, then $w$ does not have a neighbor in $\Psi(\mathcal{C})$ and $u$ does not have a neighbor in $\Psi\left(\mathcal{S}_{\ell}^{i} \backslash \mathcal{C}\right)$. Condition $(\mathrm{C})$ is an important condition, which is new compared to the definition in Section 3.6 The condition requires that each potential connector path is minimal, i.e., there is no potential connector of length 2 connecting $\Psi(\mathcal{C})$ to another component of $\Psi\left(\mathcal{S}_{\ell}^{i}\right)$ via only $u$ or only $w$.

From a potential connector path $P$ on graph $G$, we derive a potential connector path $\mathcal{P}$ on virtual nodes in $\mathcal{G}$ by determining the types of related internal nodes as follows: (D) If $P$ has one internal real node $w$, then for $\mathcal{P}$ we choose the virtual node of $w$ in layer $\ell+1$ in $\mathcal{G}$ with type 1 . (E) If $P$ has two internal real nodes $w_{1}$ and $w_{2}$, where $w_{1}$ is adjacent to $\Psi(\mathcal{C})$ and $w_{2}$ is adjacent to $\Psi\left(\mathcal{S}_{\ell}^{i} \backslash \mathcal{C}\right)$, then for $\mathcal{P}$ we choose the virtual nodes of $w_{1}$ and $w_{2}$ in layer $\ell+1$ with types 2 and 3 , respectively. Finally, for each endpoint $w$ of $P$ we add the copy of $w$ in $\mathcal{S}_{\ell}^{i}$ to $\mathcal{P}$.

\footnotetext{
${ }^{6}$ We could add condition $(C)$ to the definition of potential connector paths in Section 3 as well, but it is not necessary there.
} 


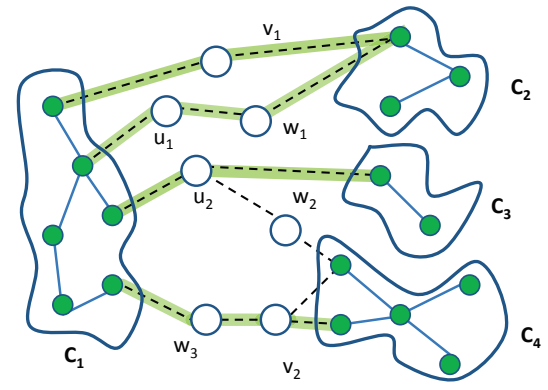

Projection of components of old nodes of class $i$ onto graph $\mathrm{G}$ and its potential connector paths

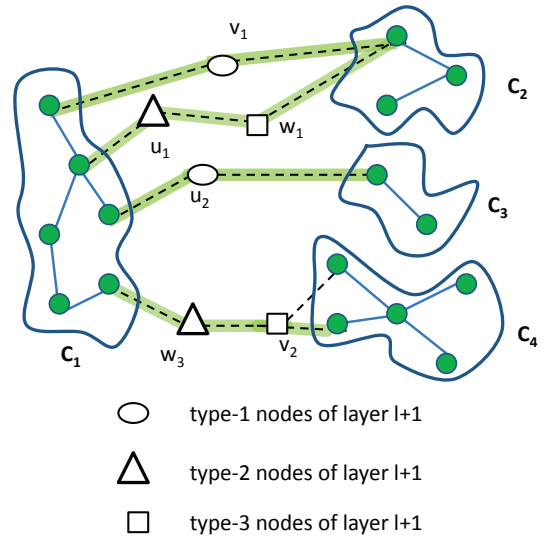

Figure 2: Potential Connector Paths for component $\mathcal{C}_{1}$ in layer $\ell+1$ copies of $G$

A given potential connector path $\mathcal{P}$ on the virtual nodes of layer $\ell+1$ is called a connector path if and only if the internal nodes of $\mathcal{P}$ are sampled. We call a connector path that has one internal node a short connector path, whereas a connector path with two internal nodes is called a long connector path.

Because of condition (C), and rules (D) and (E) above, we get the following important fact:

Proposition 4.2. For each class $i$, each type-2 virtual node $u$ of layer $\ell+1$ is on connector paths of at most one connected component of $\mathcal{G}\left[\mathcal{S}_{\ell}^{i}\right]$.

Figure 2 demonstrates an example of potential connector paths for a component $\mathcal{C}_{1} \in \mathcal{G}\left[\mathcal{S}_{\ell}^{i}\right]$. The figure on the left shows graph $G$, where the projection $\Psi\left(\mathcal{S}_{\ell}^{i}\right)$ is indicated via green nodes, and the green paths are potential connector paths of $\Psi\left(\mathcal{C}_{1}\right)$. On the right side, we see the same potential connector paths, where the type of the related internal nodes are determined according to rules (D) and (E) above, and nodes of different types are distinguished via different shapes (for clarity, virtual nodes of other types are omitted from the figure).

The following lemma shows that each component that is not alone in its class is likely to have many connector paths. The proof is similar to that of Lemma 3.3, and is deferred to Appendix D.

Lemma 4.3 (Connector Abundance Lemma). Consider a layer $\ell \geq L / 2$ and a class $i$ such that $\mathcal{S}_{L / 2}^{i} \subseteq \mathcal{S}_{\ell}^{i}$ is a dominating set of $\mathcal{G}$ and $N_{\ell}^{i} \geq 2$. Further consider an arbitrary connected component $\mathcal{C}$ of $\mathcal{G}\left[\mathcal{S}_{\ell}^{i}\right]$. Then, with probability at least $1 / 2, \mathcal{C}$ has at least $t$ internally vertex-disjoint connector paths.

\subsection{Recursive Step of Class Assignment}

From Lemma 4.3 we know that for each connected component of each class $i$ with $N_{\ell}^{i} \geq 1$, with probability at least $1 / 2$, this component has at least $t$ connector paths. For each such component, pick exactly $t$ of its connector paths. Using Markov's inequality, we get that with probability at least $1 / 4$, we have at least $M_{\ell} \cdot t$ connector paths in total, over all the classes and components. We use these connector paths to assign the class numbers of nodes of layer $\ell+1$. This part is done in a greedy fashion, in three stages as follows:

(I) For each type- 1 new node $v$ : For each class $i$, define the class- $i$ degree of $v$ to be the number of connected components of class $i$ which have a short connector path through $v$. Let $\Delta$ be the maximum class- $i$ degree of $v$ as $i$ ranges over all classes, and let $i^{*}$ be a class that attains this maximum. If $\Delta \geq 1$ : Assign node $v$ to class $i^{*}$. Also, remove all connector paths of all classes that go through $v$. Moreover, remove all connector paths of all the connected components of class $i^{*}$ which have $v$ on their short connector paths.

(II) For each type-3 new node $u$ : For each class $i$, define the class- $i$ degree of $u$ to be the number of connected components of class $i$ which have a long connector path through $u$. Let $\Delta$ be the maximum class- $i$ degree of $u$ as $i$ ranges over all classes and let $i^{*}$ be a class that attains this maximum. If $\Delta \geq 1$ : 
Assign $u$ to class $i^{*}$. Moreover, each of the $\Delta$ long connector paths of class $i^{*}$ that passes through $u$ also has a type- 2 internal node. Let these type- 2 nodes be $v_{1}, \ldots, v_{\Delta}$. We assign the nodes $v_{1}, \ldots, v_{\Delta}$ to class $i^{*}$. Then, all the connector paths that go through $u$ or any of the nodes $v_{1}, \ldots, v_{\Delta}$ are removed. We also remove all connector paths of each component of class $i^{*}$ that has a connector path going through $u$.

(III) Assign each remaining new node to a random class.

Lemma 4.4 (Fast Merger Lemma). For each $\ell \geq \frac{L}{2}$ and every assignment of the sampled layer $1, \ldots, \ell$ nodes to classes such that for all classes $i, \mathcal{S}_{L / 2}^{i}$ is a dominating set of $\mathcal{G}$, with probability at least $1 / 4$, $M_{\ell+1} \leq \frac{5}{6} \cdot M_{\ell}$

The proof is based on an accounting argument that uses the total number of remaining connector paths over all classes and components as the budget, and shows that number of components that are merged, each with at least one other component, is large. We defer the details to Appendix D.

\section{Construction of a CDS Partition}

Theorem 1.2. (restated and rephrased) Every k-vertex-connecgted graph $G$ has a CDS partition of size $\Omega\left(\frac{k}{\log ^{5} n}\right)$, and if $k=\Omega(\sqrt{n})$, then $G$ has a CDS partition of size $\Omega\left(\frac{k}{\log ^{2} n}\right)$. Moreover, such CDS partitions can be found in polynomial time.

In order to achieve this CDS partition, we use an algorithm in the style of the CDS packing of Theorem 1.4. Here, we explain the key changes: since in a CDS partition, each node can only join one CDS, we cannot use the layering style of Theorem 1.4, which uses $\Theta(\log n)$ copies of $G$ and where each node can join $O(\log n)$ CDSs. Instead, we use random layering: each node chooses a random layer number in $\{1, \ldots, L\}$ and a random type number in $\{1,2,3\}$. The construction is again recursive, with first assigning nodes of layer 1 randomly to one of $t$ random classes. This suffices to give domination. Here, $t=\delta \frac{k}{\log ^{2} n}$ if $k=\Omega(\sqrt{n})$ and $t=\delta \frac{k}{\log ^{5} n}$, otherwise. After that, for each $\ell \geq 2$, we assign class numbers of nodes of layer $\ell+1$ based on the configuration of classes in layers 1 to $\ell$, using the same greedy algorithm as in Section 4.3. Next, we re-define the connector paths, incorporating the random layering.

Connector Paths for CDS Partition: Let $V_{\ell}^{i}$ be the set of all nodes of layers 1 to $\ell$ in class $i$. Consider a component $\mathcal{C}$ of $G\left[V_{\ell}^{i}\right]$. Define potential connector paths on $G$ as in Section 4.2 (conditions (A) to (C)). Then, for each potential connector path on $G$, this path is called a connector path if its internal nodes are in layer $\ell+1$ and the types of its internal nodes satisfy rules (D) and (E) in Section 4.2 .

The main technical change, with respect to the CDS packing of Section 4, appears in obtaining a Connector Abundance Lemma, which we present in two versions, depending on the magnitude of vertex-connectivity.

Lemma 5.1 (Connector Abundance Lemma). For each class $i$ and layer $\ell \leq L / 2$ such that $N_{\ell}^{i} \geq 2$, for each connected component $\mathcal{C}$ of $G\left[V_{\ell}^{i}\right]$, with probability at least $1 / 2, \mathcal{C}$ has at least $\Omega\left(\frac{k}{\log ^{5} n}\right)$ internally vertex-disjoint connector paths, with independence between different layers $\ell \leq L / 2$.

Lemma 5.2 (Stronger Connector Abundance Lemma for Large Vertex Connectivity). Assume $k=$ $\Omega(\sqrt{n})$. W.h.p., for each class $i$ and layer $\ell \leq L / 2$ such that $N_{\ell}^{i} \geq 2$, each connected component $\mathcal{C}$ of $G\left[V_{\ell}^{i}\right]$ has at least $\Omega\left(\frac{k}{\log ^{2} n}\right)$ internally vertex-disjoint connector paths.

The proof of Lemma 5.1 is relatively straightforward application of Theorem 1.4 .

Proof of Lemma 5.1. Let $W_{\ell}^{*}$ be the set of all nodes with a layer number in $\{\ell+1, \ldots, L\}$. Since the probability of each node to be in $V_{\ell}^{*}$ is at least $1 / 2$ (because $\ell \leq L / 2$ ), Theorem 1.4 shows that, w.h.p, the vertexconnectivity of $G\left[W_{\ell}^{*}\right]$ is $\Omega\left(\frac{k}{\log ^{3} n}\right)$. It is easy to see that therefore, the vertex-connectivity of $G\left[W_{\ell}^{*} \cup S_{\ell}^{i}\right]$ is 
also $\Omega\left(\frac{k}{\log ^{3} n}\right)$. Thus, for each component $\mathcal{C}$ of $G\left[V_{\ell}^{i}\right]$, we can follow the proof of Lemma 3.3, this time using Menger's theorem on $G\left[W_{\ell}^{*} \cup S_{\ell}^{i}\right]$, and find $\Omega\left(\frac{k}{\log ^{3} n}\right)$ internally vertex-disjoint potential connector paths in graph $G\left[W_{\ell}^{*} \cup V_{\ell}^{i}\right]$. It is clear that the internal nodes of these potential connector paths are not in $V_{\ell}^{i}$, which means they are in $W_{\ell}^{*}$. For each potential connector path, for each of its internal nodes, given that this node is in $W_{\ell}^{*}$, the probability that the node is in layer $\ell+1$ and has the type which satisfies rules (A) and (B) of Section 4.2 is at least $\Theta(1 / L)=\Theta(1 / \log n)$. Hence, the probability of each of these potential connector paths being a connector path is at least $\Theta\left(1 / \log ^{2} n\right)$. From internally vertex-disjointedness of the potential connector paths, and since there are $\Omega\left(\frac{k}{\log ^{3} n}\right)$ of them, it follows that with probability at least $1 / 2$, component $\mathcal{C}$ has at least $\Omega\left(\frac{k}{\log ^{5} n}\right)$ internally vertex-disjoint connector paths.

The proof of Lemma 5.2 is more involved. Intuitively, for each class $i$, it first contracts components of $G\left[V_{1}^{i}\right]$ and then argues about all $2^{O(n / k)}$ cuts of the resulting graph, using the fact that the large vertex connectivity $k=\Omega(\sqrt{n})$ gives a good enough concentration to compensate for this large number of cuts. This proof is deferred to Appendix $\mathrm{E}$.

\section{Concluding Remarks}

In this paper, we introduce CDS partition and CDS packing as a new way of looking at the structure of graphs with a given vertex connectivity $k$. We argue that CDS packing and CDS partition can be viewed as the "vertex world" counterparts of the well-known edge-disjoint spanning trees (often attributed to 1961 works of Nash-Williams [24] and Tutte [26]). We show that each $k$-vertex-connected graph with $n$ nodes has a CDS packing of size $\Omega(k / \log n)$ and that this is existentially optimal. We also show that any such graph has a CDS partition of size $\Omega\left(k / \log ^{5} n\right)$ and if $k=\Omega(\sqrt{n})$, it has a CDS partition of size $\Omega\left(k / \log ^{2} n\right)$.

Using the new perspective provided by CDS packing, we manage to break the barrier of facing exponentially many small vertex cuts and prove that after sampling vertices with probability $p$, the remaining vertex connectivity is $\tilde{\Omega}\left(k p^{2}\right)$, w.h.p. We also explain that CDS packing is useful in networking as it provides a backbone for store-and-forward algorithms with optimal throughput. In a subsequent work [4], we show that a CDS packing of size $O(k / \log n)$ can also be computed efficiently by a distributed algorithm with time complexity $\tilde{O}(n / k)$.

Our work leaves a number of interesting and important open questions. First of all, most of our results are only tight up to logarithmic factors and it would certainly be interesting to try to close these gaps. When sampling each node with probability $p$, assuming that $k p^{2}$ is large enough, we suspect that the remaining vertex connectivity should be $\Theta\left(k p^{2}\right)$. We also believe that construction from Section 5 to compute a CDS partition should actually allow to obtain a CDS partition of size $\Omega\left(k / \log ^{2} n\right)$ for all values of $k$ and not just for $k=\Omega(\sqrt{n})$. Note that our approach would immediately give this if we could prove that when sampling with probability $1 / 2$, the remaining vertex connectivity is still $\Omega(k)$ rather than $\Omega\left(k / \log ^{3} n\right)$ as we show. Note that even proving an $\Omega\left(k / \log ^{2} n\right)$ lower bound on the size of a maximum CDS partition would still leave a logarithmic gap w.r.t. the upper bound of Theorem 1.3. Finally, we hope that CDS packings and partitions can be used to approach other problems related to vertex connectivity. In particular, if true, the approach might help to show that the sizes all vertex cuts remain around their expectations after vertex sampling. We believe that such a result would be a major contribution.

\section{Acknowledgment}

We are grateful to David Karger for helpful discussions and we also thank Bernhard Haeupler for valuable comments. 


\section{References}

[1] A. Agarwal and M. Charikar. On the advantage of network coding for improving network throughput. In Proc. IEEE Inf. Theory Workshop, pages 247-249, 2004.

[2] N. Alon and J. Spencer. The Probabilistic Method. John Wiley \& Sons, New York, 1992.

[3] A. A. Benczúr and D. R. Karger. Approximating s-t minimum cuts in $\tilde{O}\left(n^{2}\right)$ time. In Proc. 28th ACM Symp. on Theory of Computing (STOC), pages 47-55, 1996.

[4] K. Censor-Hillel, M. Ghaffari, and F. Kuhn. Distributed connected dominating set packing. Unpublished Manuscript, 2013.

[5] U. Feige, M. Halldórsson, G. Kortsarz, and A. Srinivasan. Approximating the domatic number. SIAM J. Computing, 32(1):172-195, 2002.

[6] A. Goel and S. Khanna. On the network coding advantage for wireless multicast in euclidean space. In Proc. 7th Conf. on Inf. Processing in Sensor Netw. (IPSN), pages 64-69, 2008.

[7] B. Haeupler. Analyzing network coding gossip made easy. In Proc. 43rd ACM Symp. on Theory of Computing (STOC), pages 293-302, 2011.

[8] B. L. Hartnell and D. F. Rall. Connected domatic number in planar graphs. Czech. Math. Journal, 51:173-179, 2001.

[9] S. T. Hedetniemi and R. Laskar. Connected domination in graphs. Technical Report 414, Clemson University, SC, USA, 1983.

[10] S. T. Hedetniemi and R. Laskar. Topics on domination. Annals of Discrete Mathematics, 48, 1991.

[11] A. Kanevsky. On the number of minimum size separating vertex sets in a graph and how to find all of them. In Proc. 1st ACM-SIAM Symp. on Discrete Algorithms (SODA), pages 411-421, 1990.

[12] D. R. Karger. Global min-cuts in RNC, and other ramifications of a simple min-out algorithm. In Proc. 4th ACM-SIAM Symp. on Discrete Algorithms (SODA), pages 21-30, 1993.

[13] D. R. Karger. Random sampling in cut, flow, and network design problems. In Proc. 26th ACM Symp. on Theory of Computing (STOC), pages 648-657, 1994.

[14] D. R. Karger. Using randomized sparsification to approximate minimum cuts. In Proc. 5th ACM-SIAM Symp. on Discrete Algorithms (SODA), pages 424-432, 1994.

[15] D. R. Karger. A randomized fully polynomial time approximation scheme for the all terminal network reliability problem. In Proc. 27th ACM Symp. on Theory of Computing (STOC), pages 11-17, 1995.

[16] D. R. Karger. Minimum cuts in near-linear time. In Proc. 28th ACM Symp. on Theory of Computing (STOC), pages 56-63, 1996.

[17] D. R. Karger and M. S. Levine. Finding maximum flows in undirected graphs seems easier than bipartite matching. In Proc. 30th ACM Symp. on Theory of Computing (STOC), pages 69-78, 1998.

[18] F. Kuhn, N. Lynch, and C. Newport. The abstract mac layer. Distributed Computing, 24(3):187-206, 2011.

[19] S. Kundu. Bounds on the number of disjoint spanning trees. J. of Comb. Theory, Series B, 17(2):199-203, 1974.

[20] M. Langberg and M. Médard. On the multiple unicast network coding conjecture. In Proc. 47th Allerton Conf. on Comm., Control, and Computing, pages 222-227, 2009.

[21] Z. Li, B. Li, and L. C. Lau. A constant bound on throughput improvement of multicast network coding in undirected networks. IEEE Trans. Inf. Theory, 55(3):1016-1026, 2009.

[22] M. V. Lomonosov and V. P. Polesskii. Lower bound of network reliability. Problems of Information Transmission, 7:118-123, 1971.

[23] R. Misra and C. Mandal. Rotation of CDS via connected domatic partition in ad hoc sensor networks. IEEE Trans. on Mobile Computing, 8(4):488-499, 2009.

[24] C. S. J. A. Nash-Williams. Edge-disjoint spanning trees of finite graphs. J. of the London Math. Society, 36:445$450,1961$. 
[25] P. Sanders, S. Egner, and L. Tolhuizen. Polynomial time algorithms for network information flow. In Proc. 15th ACM Symp. on Parallel Algorithms and Architectures (SPAA), pages 286-294, 2003.

[26] W. T. Tutte. On the problem of decomposing a graph into $n$ connected factors. J. of the London Math. Society, 36:221-230, 1961.

[27] B. Zelinka. Connected domatic number of a graph. Math. Slovaca, 36:387-392, 1986.

\section{A Upper Bounding the Size of CDS-Packing}

In this section, we first present the missing proof of Proposition 2.4, and then, in the more interesting part, prove Theorem 1.3, which shows that in some graphs, the maximum CDS packing size is a $\Theta(\log n)$ factor smaller than the vertex connectivity.

Proof of Proposition 2.4. Consider a vertex cut $\mathcal{C} \subseteq V$ of $G$ that has size exactly $k$. Each CDS $\tau$ must include at least one node in $\mathcal{C}$. For each $\operatorname{CDS} \tau \in C D S(G)$, pick one node $v \in C$ as a representative of $\tau$ in the cut and let us denote it by $\operatorname{Rep}(\tau)$. Thus, for any CDS-Packing of $G$, we have

$$
\sum_{\tau \in C D S(G)} x_{\tau}=\sum_{v \in \mathcal{C}} \sum_{\substack{\tau \in C D S(G) \\ \text { s.t. } v=\operatorname{Rep}(\tau)}} x_{\tau} \leq \sum_{v \in \mathcal{C}} 1=|\mathcal{C}|=k .
$$

Since the above holds for any CDS-Packing of $G$, we get that $K_{C D S}^{\prime}(G) \leq k$.

Let us recall the statement of Theorem 1.3

Theorem 1.3. (restated) For any large enough $n$ and any $k \geq 1$, there exists an $n$-node graph $G$ with vertex connectivity $k$ such that $K_{C D S}^{\prime}(G)=O\left(\frac{k}{\log n}+1\right)$.

To prove this theorem, in Lemma A.1, we present a graph $\mathrm{H}$ with vertex connectivity $k$, size between $2^{k}$ and $4^{k}$, and $K_{C D S}^{\prime}(\mathrm{H})<2$. This lemma proves the theorem for $k=O(\log n)$. To prove the theorem for the case of larger vertex-connectivity compared to $n$, in Lemma A.2, we look at randomly chosen sub-graphs of $\mathrm{H}$ and apply the probabilistic method [2].

Lemma A.1. For any $k$, there exists an n-node graph $\mathrm{H}$ with vertex connectivity $k$ and $n \in\left[2^{k}, 4^{k}\right]$ such that $K_{C D S}^{\prime}(\mathrm{H})<2$.

Proof. We obtain graph $\mathrm{H}$ by simple modifications to the graph presented by Sanders et al. [25] for proving an $\Omega(\log n)$ network coding gap in the model where each node can send distinct unit-size messages to its different neighbors.

The graph $\mathrm{H}$ has two layers. The first layer is a clique of $2 k$ nodes. The second layer has $\left(\begin{array}{c}2 k \\ k\end{array}\right)$ nodes, one for each subset of size $k$ of the nodes of the first layer. Each second layer node is connected to the $k$ first-layer nodes of the corresponding subset. Note that the total number of nodes is $\left(\begin{array}{c}2 k \\ k\end{array}\right)+2 k \in\left[2^{k}, 4^{k}\right]$. Let $\mathcal{A}$ and $\mathcal{B}$ denote the set of nodes in the first and second layer, respectively.

First, we show that $\mathrm{H}$ has vertex connectivity $k$. Since the degree of each second layer node is exactly $k$, it is clear that the vertex connectivity of $\mathrm{H}$ is at most $k$. To prove that the vertex connectivity of $\mathrm{H}$ is at least $k$, let $u$ and $v$ be two arbitrary nodes of $\mathrm{H}$. We show that there are at least $k$ internally vertex disjoint paths between $u$ and $v$. If $u$ and $v$ are both in $\mathcal{A}$, then there is one direct edge between $v$ and $u$ and there are $2 k-2$ paths of length 2 between them. If exactly one of $v$ and $u$ is in $\mathcal{A}$, e.g., suppose $u \in \mathcal{A}$ and $v \in \mathcal{B}$, then $u$ is directly connected to $k$ neighbors of $v$. Otherwise, if both $u$ and $v$ are in $\mathcal{B}$, then let $p$ be the size of the intersection of the neighbors of $v$ and $u$. Note that these neighbors are all in $\mathcal{A}$. It is clear that $u$ and $v$ have exactly $p$ paths of length 2 between themselves and $k-p$ paths of lengths 3 , and that these paths are internally vertex disjoint. 
To see that $K_{C D S}^{\prime}(H)<2$, first note that each $\operatorname{CDS} \tau$ must include at least $k+1$ nodes of $\mathcal{A}$. This is because, otherwise, there are at least $k$ nodes of $\mathcal{A}$ that are not included in $\tau$ and thus, there is a node in $\mathcal{B}$ - corresponding to a subset of size $k$ of these uncovered nodes of $\mathcal{A}$-which is not dominated by $\tau$. Thus we have,

$$
\sum_{v \in \mathcal{A}} \sum_{\substack{\tau \in C D S(\mathrm{H}) \\ \text { s.t. } v \in \tau}} x_{\tau} \geq(k+1) \cdot \sum_{\tau \in C D S(\mathrm{H})} x_{\tau} .
$$

On the other hand we have,

$$
\sum_{v \in \mathcal{A}} \sum_{\substack{\tau \in C D S(\mathrm{H}) \\ \text { s.t. } v \in \tau}} x_{\tau} \leq \sum_{v \in \mathcal{A}} 1=|\mathcal{A}|=2 k
$$

and thus we can conclude that $\sum_{\tau \in C D S(\mathrm{H})} x_{\tau} \leq \frac{2 k}{k+1}<2$. Since this holds for any CDS-Packing of $\mathrm{H}$, we get $K_{C D S}^{\prime}(H)<2$.

Note that in the above construction, we have $K_{C D S}(\mathrm{H})=1$ as $\mathrm{H}$ is connected and $K_{C D S}(\mathrm{H})$ has to be an integer.

Lemma A.2. For each large enough $k$ and $\eta \in\left[4 k, 2^{k}\right]$, there exists a sub-graph $H^{\prime} \subseteq \mathrm{H}$ that has $\eta$ nodes and vertex connectivity $k$ but $K_{C D S}^{\prime}\left(H^{\prime}\right)=O\left(\frac{k}{\log \eta}\right)$.

Proof. Pick an arbitrary $k \geq 64$, fix an $\eta \in\left[4 k, 2^{k}\right]$ and let $\beta=\frac{\log \eta}{8}$. Consider a random subset $V_{z} \subseteq V$, where $V_{z}$ includes all nodes of $\mathcal{A}$ and for each node $u \in \mathcal{B}, u$ is independently included in $V_{z}$ with probability $p$, where

$$
p=\frac{65 \beta^{2}}{\left(\begin{array}{c}
2 k-\beta \\
k
\end{array}\right)} .
$$

We now look at the sub-graph $H_{z}$ of $\mathrm{H}$ induced on $V_{z}$. With the same argument as for $\mathrm{H}$, we get that for any such $V_{z}$, the graph $H_{z}$ has vertex connectivity exactly $k$. We show that (a) with probability at least $\frac{1}{2}, V_{z}$ is such that $K_{C D S}^{\prime}\left(H_{z}\right)<\frac{2 k}{\beta}=O\left(\frac{k}{\log \eta}\right)$, and (b) with probability at least $\frac{3}{4}$, we have $\left|V_{z}\right| \leq \eta$. A union bound then completes the proof.

Property (a) We first show that with probability at least $\frac{1}{2}, V_{z}$ is such that there does not exist a subset of size $\beta$ of the nodes of $\mathcal{A}$ that dominates $V_{z}$. For each subset $W \subset \mathcal{A}$ such that $|W|=\beta$, there are $\left(\begin{array}{c}2 k-\beta \\ k\end{array}\right)$ nodes in $\mathcal{B}$ which are not dominated by $W$. Thus, for $W$ to dominate $V_{z}$, none of these second layer nodes should be included in $V_{z}$. The probability for this to happen is

$$
(1-p)^{\left(\begin{array}{c}
2 k-\beta \\
k
\end{array}\right)} \leq e^{-65 \beta^{2}}
$$

There are $\left(\begin{array}{c}2 k \\ \beta\end{array}\right)$ possibilities for set $W$. Hence, using a union bound, the probability that there exists such a set $W$ that dominates $V_{z}$ is at most

$$
\begin{aligned}
e^{-65 \beta^{2}}\left(\begin{array}{c}
2 k \\
\beta
\end{array}\right) \leq e^{-65 \beta^{2}} \cdot\left(\frac{2 e k}{\beta}\right)^{\beta} & \stackrel{(\dagger)}{<} e^{-65 \beta^{2}} \cdot\left(\eta^{2}\right)^{\beta} \\
& =e^{-65 \beta^{2}+64 \beta^{2}} \leq \frac{1}{2},
\end{aligned}
$$

where Inequality $(\dagger)$ follows since $64 \leq k \leq \frac{\eta}{4}$, which gives $2 e k<k^{2}<\eta^{2}$.

Thus, with probability at least $\frac{1}{2}, V_{z}$ is such that each CDS of $H_{z}$ includes at least $\beta+1$ nodes of $\mathcal{A}$. From this, similar to the last part of the proof of Lemma A.1, we have that, $\sum_{\tau \in C D S(\mathrm{H})} x_{\tau} \leq \frac{2 k}{\beta+1}<\frac{2 k}{\beta}$. Since this holds for any packing of $H_{z}$, we get that with probability at least $\frac{1}{2}, V_{z}$ is such that $K_{C D S}^{\prime}\left(H_{z}\right)<\frac{2 k}{\beta}$. 
Property (b) Note that $\mathbb{E}\left[\left|V_{z}\right|\right]=2 k+p \cdot\left(\begin{array}{c}2 k \\ k\end{array}\right)$. Substituting $p=\frac{65 \beta^{2}}{\left(\begin{array}{c}2 k-\beta \\ k\end{array}\right)}$ and noting that $\beta \leq \frac{k}{2}$, we get

$$
\begin{aligned}
\mathbb{E}\left[\left|V_{z}\right|\right]-2 k & =p \cdot\left(\begin{array}{c}
2 k \\
k
\end{array}\right)=65 \beta^{2} \cdot \frac{\left(\begin{array}{c}
2 k \\
k
\end{array}\right)}{\left(\begin{array}{c}
2 k-\beta \\
k
\end{array}\right)} \\
& =65 \beta^{2} \cdot \frac{2 k}{2 k-\beta} \cdot \frac{2 k-1}{2 k-\beta-1} \cdots \frac{k+1}{k-\beta+1} \\
& \leq 65 \beta^{2} \cdot\left(1+\frac{2 \beta}{k}\right)^{k} \\
& \leq \frac{65 \log ^{2} \eta}{64} \cdot \eta^{\frac{1}{4}} \leq \frac{\eta}{4} .
\end{aligned}
$$

As the second-layer nodes are picked independently, for $\eta$ sufficiently large, we can apply a Chernoff bound to get $\operatorname{Pr}\left[\left|V_{z}\right|-2 k>\frac{\eta}{2}\right] \leq \frac{1}{4}$. Since $2 k \leq \frac{\eta}{2}$, we then obtain $\operatorname{Pr}\left[\left|V_{z}\right|>\eta\right] \leq \frac{1}{4}$. If desired, we can adjust the number of nodes to exactly $\eta$ by adding enough nodes in the second layer which are each connected to all nodes of the first layer.

\section{B Missing Proof of Section 2.3: CDS Packing vs. Throughput}

In this section, we prove Theorem 2.5. For simplicity, we restate the theorem.

Theorem 2.5. (restated) A CDS packing with size t provides a store-and-forward backbone with broadcast throughput $\Omega(t)$ messages per round. Inversely, a store-and-forward broadcast algorithm with throughput $t$ messages per round induces a CDS packing of size at least $t$.

Proof of Theorem 2.5. First consider a CDS $\tau$ and suppose that the graph induced by $\tau$ has diameter $D_{\tau}$. Using $\tau$, we can perform $p$ broadcasts (or multicast or unicasts) in time $O\left(p+D_{\tau}\right)$. This can be seen as follows: Since $\tau$ is a dominating set, we can deliver each message to a node of $\tau$ in at most $p$ rounds. Because $\tau$ is connected, $O\left(p+D_{\tau}\right)$ rounds are enough to broadcast the messages to all nodes in $\tau$. Finally, because $\tau$ is a dominating set, at most $p$ more rounds are enough to deliver the messages to all the desired destination nodes. Hence, a CDS structure allows for performing broadcasts with an (amortized) rate of $\Omega(1)$ messages per round. In other words, a CDS can be viewed as a communication backbone with throughout $\Omega(1)$ messages per round.

Consequently, $t$ vertex-disjoint CDS sets form a communication backbone with throughput of $\Omega(t)$ messages per round. Intuitively, we can use those $t$ vertex-disjoint sets in parallel with each other and get throughput of $\Omega(1)$ message per round from each of them. For a more formal description, consider $p$ broadcasts such that no more than $q$ broadcasts have the same source node. We first deliver each messages to a randomly and uniformly chosen CDS set. This can be done in time at most $q$. With high probability, the number of messages in each CDS is $O\left(\frac{p}{t}+\log n\right)$ and thus, we can simultaneously broadcast messages in time $O\left(\frac{p}{t}+\log n+D_{\max }\right)$ where $D_{\max }$ is the maximum diameter of the CDSs. Thus, the total time for completing all the broadcasts is $O\left(q+\frac{p}{t}+\log n+D_{\max }\right)$. That is, we can perform the broadcasts with a rate (throughput) of $\Omega(\min \{t, p / q\})$. Note that since each source can only send one packet per round, if $q \leq t$, then the maximum achievable throughout with any algorithm including network coding approaches is at most $q$ packets per round. In other words, in that case, the bottleneck is not the communication protocol but rather the sources of the messages. As long as no node is the source of more than $\Theta(p / t)$ messages, $t$ vertex-disjoint CDS sets form a communication backbone with throughput $\Omega(t)$.

Similarly one can see that a CDS packing with size $t$ provides a backbone with a throughput of $\Omega(t)$ messages per round. The only change with respect to above description is that now each node $v$ spends a $x_{\tau}$-fraction of its time for sending the messages assigned to CDS $\tau$ for every $\tau$ such that $v \in \tau$. Further, messages are assigned to each CDS $\tau$ with probability proportional to $x_{\tau}$. We remark here that even though this scheme provides a backbone with throughput $\Omega(t)$, if the weights $x_{\tau}$ are too small, the outlined time 
sharing might impose a considerably large additive term on the overall time for completing the broadcasts. In fact since the number of potential CDS sets can be exponential, the time sharing might lead to exponentially large additive terms. Note that in the CDS packing we present in Theorem 1.1, each CDS has weight at least $\Omega(1 / \log n)$ and thus using the partition also leads to an asymptotically optimal throughput for a relatively small number of broadcast messages.

Let us now argue that a broadcast protocol with throughput $t$ also leads to a CDS packing of size $t$. Suppose that there exists a (possibly large enough) number $p$ and a store-and-forward algorithm which broadcasts $p$ messages (originating from potentially different sources) in $T \leq \frac{p}{t}$ rounds. For each message $\sigma$ that is being broadcast, define set $S(\sigma)$ to be the set of nodes that send $\sigma$ in some round of the algorithm. Clearly $S(\sigma)$ induces a connected sub-graph and because every node needs to receive the message $S(\sigma)$ also is a dominating set. For each node $v$ and message $\sigma$, let $N_{\sigma}(v)$ be the number of rounds in which node $v$ sends message $\sigma$ and let $y_{\sigma}(v)=\frac{N_{\sigma}(v)}{T}$. Moreover, for each $\operatorname{CDS} \tau$ such that $v \in \tau$, let

$$
z_{\tau}(v)=\sum_{\substack{\sigma \\ S(\sigma)=\tau \wedge v \in \tau}} y_{\tau}(v)
$$

Finally, let $x_{\tau}=\min _{v \in \tau}\left\{z_{\tau}(v)\right\}$. Given these parameters, first notice that for each node $v$, we have

$$
\sum_{\substack{\tau \\ v \in \tau}} x_{\tau} \leq \sum_{\substack{\tau \\ v \in \tau}} z_{\tau}(v)=\sum_{\substack{\tau \\ v \in \tau}} \sum_{\substack{\sigma \\ S(\sigma)=\tau}} y_{\sigma}(v)=\sum_{\sigma} \frac{N_{\sigma}(v)}{T} \stackrel{(\dagger)}{\leq} 1 .
$$

Here, Inequality ( $\dagger$ ) is because in each round, node $v$ can send at most one message and thus, $\sum_{\sigma} N_{\sigma}(v) \leq T$. On the other hand, we show that $\sum_{\tau} x_{\tau} \geq \frac{p}{T}=\Omega(t)$. For this purpose, consider a CDS $\tau$ and let $u^{*}$ be a node such that $z_{\tau}\left(u^{*}\right)=x_{\tau}$. Since each message $\sigma$ such that $S(\sigma)=\tau$ is sent at least once by $u^{*}$, we have

$$
\begin{aligned}
\sum_{\substack{\sigma \\
S(\sigma)=\tau}} 1 \leq \sum_{\substack{\sigma \\
S(\sigma)=\tau}} N_{\sigma}\left(u^{*}\right) & =\sum_{\substack{\sigma \\
S(\sigma)=\tau}} y_{\sigma}\left(u^{*}\right) \cdot T \\
& =z_{\tau}\left(u^{*}\right) \cdot T=x_{\tau} \cdot T
\end{aligned}
$$

Moreover, we have that

$$
p=\sum_{\sigma} 1=\sum_{\tau} \sum_{\substack{\sigma \\ S(\sigma)=\tau}} 1 \leq \sum_{\tau} x_{\tau} \cdot T
$$

Thus, $\sum_{\tau} x_{\tau} \geq \frac{p}{T}$. Since $T \leq \frac{p}{t}$, we get that $\sum_{\tau} x_{\tau} \geq t$.

\section{Missing Proofs of Section 3}

Proof of Lemma 3.1. Note that since $G$ has vertex connectivity $k$, each node $v \in V$ has at least $k$ neighbors. Therefore, each virtual node $\nu \in \mathcal{V}$ has in expectation at least $\frac{k p L}{2 L}=\alpha \sqrt{k} \log n / 2=\Omega(\log n)$ virtual neighbors in $\mathcal{S}_{\frac{L}{2}}$. For every $\lambda>0$ and a sufficiently large $\alpha>0$, a standard Chernoff argument combined with union bound over all virtual nodes implies that each node has at least one neighbor in $\mathcal{S}_{\frac{L}{2}}$, w.h.p.

Proof of Lemma 3.2. Assume $\mathcal{S}_{L / 2} \in \mathcal{D}$. Then, for the first part of lemma, note that since $\mathcal{S}_{L / 2} \in \mathcal{D}$ and for each layer $\ell \geq L / 2, \mathcal{S}_{\frac{L}{2}} \subseteq S_{\ell}$, we get that $S_{\ell}$ dominates set $\mathcal{V}$. Thus, each virtual sampled node in layer $\ell+1$ has a neighbor in $S_{\ell}$ which means that each connected component of $\mathcal{G}\left[\mathcal{S}_{\ell+1}\right]$ contains at least one connected component of $\mathcal{G}\left[\mathcal{S}_{\ell}\right]$. Hence, $N_{\ell+1} \leq N_{\ell}$, which also means that $M_{\ell+1} \leq M_{\ell}$.

For the second part of the lemma, fix an arbitrary $\mathcal{T} \in \mathcal{D}_{\ell}$ and suppose that $\mathcal{S}_{\ell}=\mathcal{T}$. Let $X$ be the number of components of $\mathcal{G}\left[\mathcal{S}_{\ell}\right]$ for which there is at least one connector path in layer $\ell+1$. Note that each such component gets merged with at least one other component. By Lemma 3.3 , the expectation of $X$ is at 
least $\mathbb{E}[X] \geq N_{\ell} / 2$. Let $q$ be the probability that $X \geq N_{\ell} / 4$. We have $\mathbb{E}[X]=\sum_{x=1}^{N_{\ell}} x \operatorname{Pr}(X=x)<$ $q \cdot N_{\ell}+(1-q) \frac{N_{\ell}}{4}$. Together with the upper bound $N_{\ell} / 2$ on $\mathbb{E}[X]$, this gives $q>1 / 3$. If $X \geq N_{\ell} / 4$, at least $1 / 4$ of the components of $\mathcal{G}\left[\mathcal{S}_{\ell}\right]$ have at least one connector path in layer $\ell+1$, i.e., are connected to some other component of $\mathcal{G}\left[\mathcal{S}_{\ell}\right]$. In that case, $N_{\ell+1} \leq \frac{7}{8} \cdot N_{\ell}$. Thus, $\operatorname{Pr}\left[M_{\ell+1} \leq \frac{9}{10} \cdot M_{\ell} \mid \mathcal{S}_{\ell}=\mathcal{T}\right] \geq 1 / 3$.

Proof of Theorem 1.5. From Lemma 3.1, we know that with high probability, $\mathcal{S}_{\frac{L}{2}}$ is a dominating set. Assuming that $\mathcal{S}_{\frac{L}{2}}$ is a dominating set, we can use Lemma 3.2. Thus, we get that with addition of sampled virtual nodes of each layer $\ell \geq L / 2$, the number of components of the sampled virtual nodes goes down by a constant factor, with probability at least $1 / 3$ and independent of previous layers. Hence, by a standard Chernoff argument, we obtain that after $L=\lambda \log n$ layers, with high probability, $\mathcal{S}$ is a connected dominating set of graph $\mathcal{G}$. Thus, $S$ is a connected dominating set of graph $G$, and hence, in particular, $G[S]$ is connected.

We conclude this section by presenting Observation 1.6 , which shows that the bound of Theorem 1.5 is optimal up to an $O(\sqrt{\log n})$ factor.

Observation 1.6. (restated) For every $k$ and $n$, there exists an $n$-node graph $G$ with vertex connectivity $k$ such that if we independently sample vertices with probability $p \leq \sqrt{\frac{\log (n / 2 k)}{2 k}}$, then the subgraph induced by the sampled nodes is disconnected with probability at least $1 / 2$.

Proof of Observation 1.6 Consider a graph $G$ composed of a chain of $\frac{n}{k}$ cliques, each of size $k$, where each two consecutive cliques on the chain are connected by a matching of size $k$. For simplicity, assume that $\frac{n}{k}$ is an even integer. Clearly, this graph has vertex connectivity $k$. Suppose that the sampling probability $p$ is less than $\sqrt{\log \left(\frac{n}{2 k}\right) /(2 k)}$. We show that, with probability at least $1 / 2$, the graph induced on the sampled vertices is disconnected.

Let us number the cliques from 1 to $\frac{n}{k}$. First note that some nodes of each clique are sampled w.h.p. Let $J=\left\{j \mid j \in\left[1, \frac{n}{k}\right] \wedge j \equiv 0(\bmod 2)\right\}$, i.e., the set of even numbers in the range $\left[1, \frac{n}{k}\right]$. If the sampled graph is connected, then for each $j \in J$, at least on of the edges of the matching connecting clique $j-1$ with clique $j$ has to be in the sampled graph. For this, there have to be two adjacent nodes $u$ from clique $j-1$ and $v$ from clique $j$ that are both sampled. We call such a pair of nodes connecting. For each $j \in J$, there are $k$ possible choices for a connecting pair and each choice has probability $\frac{1}{s^{2}}$ to be sampled (to be connecting). Moreover, these pairs are vertex disjoint. Thus, for each $j \in J$, the probability that the sampled graph has a connecting pair between cliques $j-1$ and $j$ is

$$
1-\left(1-\frac{1}{s^{2}}\right)^{k} \leq 1-4^{-k / s^{2}}<1-4^{-\log \left(\frac{n}{2 k}\right) / 2}=1-\frac{2 k}{n} .
$$

Since $J$ only contains even values, the pairs of consequent cliques that we consider are disjoint. Thus, the events whether they have a connecting pair or not are independent. Therefore, the probability that we have at least one connecting pair for each value of $j \in J$ is at most $\left(1-\frac{2 k}{n}\right)^{|J|}=\left(1-\frac{2 k}{n}\right)^{\frac{n}{2 k}}<\frac{1}{e}<1 / 2$.

\section{Missing Proofs of Section 4}

Proof of Lemma 4.1. For each node real node $v, v$ has at least $k$ real neighbors and in these $k$ real neighbors, in expectation at least $\frac{k q}{2 t}=\Omega(\log n)$ virtual nodes are sampled, have layer number 1 to $\frac{L}{2}$, and join class $i$, i.e., are in $\mathcal{S}_{\frac{L}{2}}^{i}$. Thus, the claim follows from a standard Chernoff argument combined with a union bound over all choices of $v$ and over all classes.

Proof of Lemma 4.3. As in the proof of Lemma 3.3, using Menger's Theorem on graph $G$ along with the Domination Lemma (Lemma 4.1), we can infer that if connected component $\mathcal{C}$ is not alone in its class, then $\Psi(\mathcal{C})$ has at least $k$ internally vertex-disjoint potential connector paths connecting it to $\Psi\left(\mathcal{S}_{\ell}^{i} \backslash \mathcal{C}\right)$, in graph 
$G$. Using rules (D) and (E) in Section 4.2, we get $k$ internally vertex-disjoint potential connector paths on the virtual nodes of layer $l+1$. It is clear that during the transition from the real nodes to the virtual nodes, the potential connector paths remain internally vertex-disjoint. Now, for each fixed potential connector path on the virtual nodes, the probability that the internal nodes of this path are sampled is at least $q^{2}$. Hence, in expectation, $\mathcal{C}$ has $k q^{2}$ internally vertex-disjoint connector paths (on virtual nodes). A simple application of Markov's inequality shows that with probability at least $1 / 2, \mathcal{C}$ has at least $t=\Omega\left(k q^{2}\right)$ internally vertexdisjoint connector paths.

Proof of Lemma 4.4. Consider a class $i$ such that $N_{\ell}^{i} \geq 2$ and let $\mathcal{C}_{1}$ be a connected component of $\mathcal{G}\left[\mathcal{S}_{\ell}^{i}\right]$. We say component $\mathcal{C}_{1}$ is good if for at least one connector path $p$ of $\mathcal{C}_{1}$, all internal nodes of $p$ - one or two nodes depending on whether $p$ is short or long - join class $i$. Note that if $\mathcal{C}_{1}$ is good, then it gets connected to another component of $\mathcal{G}\left[\mathcal{S}_{\ell}^{i}\right]$. In order to prove the lemma, we show that with probability at least $1 / 2$, at least $\frac{M_{\ell}}{3}$ connected components (summed up over all classes) are good. This is achieved using a simple accounting method by considering the number of remaining connector paths as the budget.

We know that with probability at least $1 / 2$, initially we have a budget of $M_{\ell} \cdot t$. We will show that the greedy algorithm spends this budget in a manner that at the end, we get $M_{\ell+1} \leq \frac{5}{6} M_{\ell}$.

In each step of each of stages I or II, if respectively a type- 1 node or a type- 3 nodes and some associated type-2 nodes join a class, then at least $\Delta$ components become good where $\Delta$ is defined as explained in the algorithm description. We show that in that case, we remove at most $3 \Delta t$ connector paths in the related bookkeeping part. Thus, in the accounting argument, we get that at most $3 \Delta \cdot t$ amount of budget is spent and $\Delta$ components become good. Hence, in total over all steps, at least $\frac{M_{\ell}}{3}$ components become good.

Let us first check the case of short connector paths, which is performed in stage I. Let $v$ be the new type-1 node under consideration in this step and suppose that the related $\Delta \geq 2$, and node $v$ joins class $i^{*}$. For class $i^{*}$, we remove all paths of all connected components of $i^{*}$ that have $v$ on their short connector paths. This includes $\Delta$ such connected components, and $t$ connector paths for each such component. Thus, in total we remove at most $\Delta \cdot t$ connector paths of components of class $i^{*}$. For each class $i \neq i^{*}$, we remove at most $\Delta$ connector paths. This is because $v$ can be on short connector paths of at most $\Delta$ components, at most once for each such component. These are respectively because of definition of $\Delta$ and due to internally vertex-disjointedness of connector paths of each component. There are less than $t$ classes other than $i^{*}$, so in total over all classes other than $i^{*}$, we remove at most $\Delta \cdot t$ connector paths. Therefore, we can conclude that the total amount of decrease in budget is at most $2 \Delta \cdot t$.

Now we check the case of long connector paths, performed in stage II. Suppose that in this step, we are working on a type- 3 new node $u$, it has $\Delta \geq 1$, and we assign node $u$ and associated type- 2 new nodes $v_{1}$, $\ldots, v_{\Delta}$ to class $i^{*}$. It follows from Proposition 4.2 that nodes $v_{1}, \ldots, v_{\Delta}$ are not on long connector paths of components of class $i^{*}$ other than the $\Delta$ components which have long paths through $u$. Thus, any connector path of class $i^{*}$ that goes through any of $v_{1}$ to $v_{\Delta}$ also goes through $u$. For each component of class $i^{*}$ that has a long connector path through $u$, we remove all the connector paths. By definition of $\Delta$, there are $\Delta$ such components and from each such component, we remove at most $t$ paths. Hence, the number of such connector paths that are removed is at most $\Delta \cdot t$. On the other hand, for each class $i \neq i^{*}$, we remove at most $2 \Delta$ connector paths. This is because by definition of $\Delta$, removing just node $u$ removes at most $\Delta$ long paths from each class. Moreover, because of Proposition 4.2 and internally vertex-disjointedness of connector paths of each component, removing each type-2 node $v_{j}$ (where $j \in\{1,2, \ldots, \Delta\}$ ) removes at most one long connector path of one connected component of class $i \neq i^{*}$. Over all classes $i \neq i^{*}$, in total we remove at most $2 \Delta \cdot t$ connector paths. Hence, when summed up with removed connector paths related to class $i^{*}$, we get that the total amount of decrease in the budget is at most $3 \Delta \cdot t$.

\section{E Missing Proofs of Section 5}

Proof of Lemma 5.2. Fix a class $i$. We start by studying the connected components of $G\left[V_{1}^{i}\right]$. Each node has probability $\frac{1}{t L}=\frac{\log n}{\delta \lambda k}$ to be in $V_{1}^{i}$. Therefore, $\mathbb{E}\left[\left|V_{1}^{i}\right|\right]=\frac{n \log n}{\delta \lambda k}$. Using a Chernoff bound we get that for 
$\delta$ sufficiently small, w.h.p., $\left|V_{1}^{i}\right| \leq \frac{2 n \log n}{\delta \lambda k}$. Moreover, since each node $v \in V$ has at least $k$ neighbors in $G$, the expected number of neighbors of $v$ in $V_{1}^{i}$ is at least $\Omega(\log n)$. Using another Chernoff bound (and $\delta$ sufficiently small) and then a union bound over all choices of $v$, we get that w.h.p., each node $v \in V$ has $\Omega(\log n)$ neighbors in $V_{1}^{i}$. Therefore, in particular, each node $v \in V_{1}^{i}$ has $\Omega(\log n)$ neighbors in $V_{1}^{i}$. In other words, w.h.p., the degree of each node in $G\left[V_{1}^{i}\right]$ is $\Omega(\log n)$. Thus, w.h.p., $G\left[V_{1}^{i}\right]$ has at $\operatorname{most} \frac{2 n \log n}{\delta \lambda k}$ nodes, each of degree $\Omega(\log n)$. Therefore, for an appropriate choice of the constant $\delta$, w.h.p., the number of connected components of $G\left[V_{1}^{i}\right]$ is at most $\varepsilon \cdot \frac{n}{k}$ for a given constant $\varepsilon>0$. Let $\Sigma^{i}$ be the set of all connected components of $G\left[V_{1}^{i}\right]$.

We call each nonempty set $A$ which is a strict subset of $\Sigma^{i}$, i.e., $A \subset \Sigma^{i}$, a component cut of $G\left[V_{1}^{i}\right]$. Since, w.h.p., we have $\left|\Sigma^{i}\right| \leq \varepsilon \frac{n}{k}$, the number of component cuts of $G\left[V_{1}^{i}\right]$ is at most $2^{\varepsilon n / k}$, w.h.p.

For each layer $\ell \in\left[2, \frac{L}{2}\right]$ and each component cut $A$ of $G\left[V_{1}^{i}\right]$, we say $A$ is $\ell$-rich if there are at least $\frac{k}{8}$ internally vertex-disjoint paths $p$ which satisfy the following conditions: (1) $p$ has one end point $s \in A$ and the other endpoint $t \in S \backslash A$, (2) $p$ has at most two internal nodes, (3) if $p$ has two internal nodes and has the form $s, u, w, t$, then $u$ does not have a neighbor in $S-A$ and $w$ does not have a neighbor in $S$, (4) all internal nodes of $p$ are in layers $[\ell+1, L]$. We first show that with high probability, for each layer $\ell \in\left[2, \frac{L}{2}\right]$ and each component cut $A$ of $G\left[V_{1}^{i}\right], A$ is $\ell$-rich.

Consider an arbitrary component cut $A$ of $G\left[V_{1}^{i}\right]$. Since graph $G$ is $k$-vertex connected and because $V_{1}^{i}$ is a dominating set (cf. Lemma 4.1), there are at least $k$ internally vertex-disjoint paths which satisfy conditions (1) and (2). The details of this argument are similar to the first part of the proof of Lemma 3.3. Each of these $k$ paths can be trimmed to also satisfy condition (3). To see this, consider a path $p$ as described in condition (3). If $v$ has a neighbor in $S \backslash A$ then there is a trimmed path $p^{\prime}$ from some node in $A$ to $v$ to some node in $S \backslash A$, which satisfies (3). Similarly, if $w$ has a neighbor in $A$, then there is a trimmed path $p^{\prime}$ from $A$ to $w$ to some node in $S \backslash A$, which satisfies (3). In either case, the trimmed path $p^{\prime}$ remains internally vertex-disjoint from the other $k-1$ paths.

Thus far, we have found $k$ internally vertex-disjoint paths of component cut $A$ satisfying conditions (1) to (3). Now, each internal node $u$ on each of these $k$ paths has probability at least $\frac{1}{2}$ to be in one of layers $[\ell+1, L]$. Formally, this is because, the layer number of $u$ is chosen randomly and so far, the only information exposed about $u$ is that it is not in $V_{1}^{i}$. Let layer $(u)$ be the layer number of $u$. We get

$$
\begin{aligned}
\operatorname{Pr}\left[\text { layer }(u) \in[\ell+1, L] \mid u \notin V_{1}^{i}\right] & =\frac{\operatorname{Pr}\left[\text { layer }(u) \in[\ell+1, L] \wedge u \notin V_{1}^{i}\right]}{\operatorname{Pr}\left[u \notin V_{1}^{i}\right]} \\
& =\frac{\operatorname{Pr}[\text { layer }(u) \in[\ell+1, L]]}{\operatorname{Pr}\left[u \notin V_{1}^{i}\right]}=\frac{\frac{L-\ell}{L}}{1-\frac{1}{L t}} \geq \frac{L-\ell}{L} \geq \frac{1}{2},
\end{aligned}
$$

where the last inequality holds because $\ell \leq \frac{L}{2}$. Hence, each internal node $u$ on each path $p$ out of the $k$ internally vertex-disjoint paths for $A$ has probability at least $1 / 2$ to be in layer $[\ell+1, L]$. The expected number of paths that also satisfy condition (4) therefore is at least $\frac{k}{4}$. Since the paths are internally vertexdisjoint and layer numbers are chosen independently, we can use a Chernoff bound and conclude that with probability at least $1-e^{-k / 32}$, at least $\frac{k}{8}$ of the paths satisfy condition (4). Consequently, with probability at least $1-e^{-k / 32}$, the component cut $A$ of $G\left[V_{1}^{i}\right]$ is $\ell$-rich.

Now there are at most $2^{\varepsilon n / k}$ component cuts for $G\left[V_{1}^{i}\right]$. Thus, using a union bound, the probability that there exists one of them that is not $\ell$-rich is at most $2^{\varepsilon n / k} \cdot e^{-k / 48}$. Since $k=\Omega(\sqrt{n})$, for $\varepsilon$ small enough, this probability is less than $2^{-\Omega(\sqrt{n})}=2^{-\omega(\log n)}$. Hence, w.h.p., each component cut of $G\left[V_{1}^{i}\right]$ is $\ell$-rich. Using a union bound over all choices of $\ell \in\left[2, \frac{L}{2}\right]$, we can also conclude that for each such $\ell$, each component cut of $G\left[V_{1}^{i}\right]$ is $\ell$-rich.

We are now ready to show that each connected component $\mathcal{C}$ of $G\left[V_{\ell}^{i}\right]$ has at least $\Omega\left(k / L^{2}\right)$ internally vertex-disjoint connector paths. Note that each component $\mathcal{C}$ is composed of a subset of the connected components of $G\left[V_{1}^{i}\right]$ and some nodes of layers $2, \ldots, \ell$. Hence, there is a component cut $A^{\prime}$ of $G\left[V_{1}^{i}\right]$ such that $\mathcal{C} \cap V_{1}^{i}=A^{\prime}$. Since the component cut $A^{\prime}$ is $\ell$-rich w.h.p., there are at least $\frac{k}{8}$ paths which satisfy conditions (1) to (4) above. Each such path $p$ satisfies conditions (A), (B). However, path $p$ might not directly satisfy 
condition (C). This is because, it is possible that $p$ is defined as $s, v, w, t$ which satisfies condition (3) but in graph $G\left[V_{\ell}^{i}\right]$, node $v$ has a neighbor in $W_{\ell}^{i}-\mathcal{C}$ or node $w$ has a neighbor in $\mathcal{C}$. Though, in either case, we can get a path $p^{\prime}$ of length 2 which satisfies conditions (A), (B), and (C) with just one internal node, either $v$ or $w$. Note that $p^{\prime}$ still remains internally-vertex-disjoint from the other paths. Moreover, the internal node of $p^{\prime}$ is in layers $[\ell+1, L]$.

So far, we have found $\frac{k}{8}$ potential connectors for $\mathcal{C}$ which have their internal nodes in layers in $[\ell+1, L]$. For each internal node $v$ of any of these paths, it holds that $v$ is in layer $\ell+1$ with probability greater than $\frac{1}{L}$. Formally this is true because, given that node $v$ is in a layer in $[\ell+1, L]$, the exact layer number of $v$ can be chosen after making all the decisions for the first $\ell$ layers. Therefore, each of the paths that we have found so far has probability at least $\frac{1}{L^{2}}$ to satisfy the corresponding rule conditions (D) or (E) such that it would be a connector path for $\mathcal{C}$. Hence, the expected number of connector paths of $\mathcal{C}$ is at least $\frac{k}{8 L^{2}}$. Since the potential connectors we found (which have internal nodes in layers $\ell+1$ to $L$ ) are internally vertex-disjoint, the events of them being connector paths for $\mathcal{C}$ are independent. Thus, using a Chernoff bound we get that w.h.p., $\mathcal{C}$ has at least $\Omega\left(k / L^{2}\right)=\Omega\left(k / \log ^{2} n\right)=\omega(\log n)$ connector paths. A union bound over all connected components of class $i$, over all layers $\ell \in\left[2, \frac{L}{2}\right]$, and over all choices of the class $i$ completes the proof. 